\title{
Prosocial behavior relates to the rate and timing of cortical thinning from adolescence to young adulthood
}

\author{
Lia Ferschmann ${ }^{\mathrm{a}, *}$, Nandita Vijayakumar ${ }^{\mathrm{b}}$, Håkon Grydeland ${ }^{\mathrm{a}}$, Knut Overbye ${ }^{\mathrm{a}}$, \\ Donatas Sederevicius ${ }^{a}$, Paulina Due-Tønnessen ${ }^{c}$, Anders M. Fjell ${ }^{\mathrm{a}}$, Kristine B. Walhovd ${ }^{\mathrm{a}}$, \\ Jennifer H. Pfeifer ${ }^{d}$, Christian K. Tamnes ${ }^{e}$ \\ ${ }^{\text {a }}$ Center for Lifespan Changes in Brain and Cognition, Department of Psychology, University of Oslo, Norway \\ ${ }^{\mathrm{b}}$ School of Psychology, Deakin University, Melbourne, Australia \\ ${ }^{\mathrm{c}}$ Department of Radiology, Rikshospitalet, Oslo University Hospital, Department of Psychology, University of Oslo, Norway \\ d Department of Psychology, University of Oregon, Eugene, USA \\ e PROMENTA Research Center, Department of Psychology, University of Oslo, Department of Psychiatry, Diakonhjemmet Hospital, NORMENT, Division of Mental \\ Health and Addiction, Oslo University Hospital \& Institute of Clinical Medicine, University of Oslo, Oslo, Norway
}

\section{A R T I C L E I N F O}

\section{Keywords:}

Adolescence

Cortical thickness

Development

Longitudinal

Prosocial behavior

Structural MRI

\begin{abstract}
A B S T R A C T
Prosocial behavior, or voluntary actions that intentionally benefit others, relate to desirable developmental outcomes such as peer acceptance, while lack of prosocial behavior has been associated with several neurodevelopmental disorders. Mapping the biological foundations of prosociality may thus aid our understanding of both normal and abnormal development, yet how prosociality relates to cortical development is largely unknown. Here, relations between prosociality, as measured by the Strengths and Difficulties Questionnaire (selfreport), and changes in thickness across the cortical mantle were examined using mixed-effects models. The sample consisted of 169 healthy individuals ( 92 females) aged 12-26 with repeated MRI from up to 3 time points, at approximately 3-year intervals (301 scans). In regions associated with social cognition and behavioral control, higher prosociality was associated with greater cortical thinning during early-to-middle adolescence, followed by attenuation of this process during the transition to young adulthood. Comparatively, lower prosociality was related to initially slower thinning, followed by comparatively protracted thinning into the mid-twenties. This study showed that prosocial behavior is associated with regional development of cortical thickness in adolescence and young adulthood. The results suggest that the rate of thinning in these regions, as well as its timing, may be factors related to prosocial behavior.
\end{abstract}

\section{Introduction}

Prosociality refers to voluntary actions that intentionally benefit others (Eisenberg et al., 2015). Basic forms of prosociality emerge early in childhood, but prosocial behaviors continue to develop throughout childhood and adolescence and into early adulthood (Eisenberg et al., 2002, 2015; Warneken, 2016). High prosociality has been associated with greater peer acceptance and academic achievement (Caprara et al., 2000), and low prosociality with several developmental disorders, e.g. conduct disorder (CD; Hay et al., 2010). Mapping the brain correlates of prosociality may thus aid our understanding of the neurobiological foundations of both normal and abnormal development.

Theoretical models suggest that prosociality may rely on several neural systems. Firstly, a major source of the increase in prosociality is development of social cognitive skills, e.g. Theory of Mind (ToM; Eisenberg et al., 2015; Kuhnert et al., 2017). Modest, but consistent associations between ToM and prosociality exist and increase in strength with age (Imuta et al., 2016). ToM also predicts prosocial behavior

\footnotetext{
* Corresponding author at: Center for Lifespan Changes in Brain and Cognition (LCBC), Department of Psychology, University of Oslo, P.O. Box 1094 Blindern, 0317 Oslo, Norway.

E-mail addresses: lia.ferschmann@psykologi.uio.no (L. Ferschmann), nvijaya@deakin.edu.au (N. Vijayakumar), hakon.grydeland@psykologi.uio.no

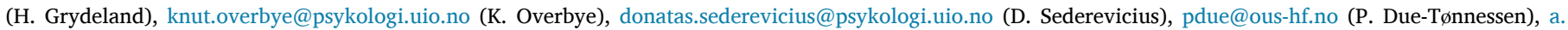

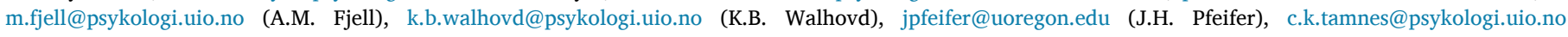
(C.K. Tamnes).
} 
prospectively (Caputi et al., 2012; Kuhnert et al., 2017). Secondly, in some situations, prosociality may be evolutionarily adaptive for functioning in social groups (Nowak, 2006). However, excessive sharing and helping can also put a strain on individual's resources and may thus be maladaptive. The costs of being prosocial must be weighed against the potential benefits, a process requiring value-based decision-making and behavioral control (Steinbeis, 2018). Third, adaptive prosociality may depend on the dynamic interplay of socio-cognitive (e.g. ToM) and socio-affective processes (e.g. empathy, compassion; Preckel et al., 2018; Tusche et al., 2016).

Functional and structural imaging studies support these theoretical models. From childhood to adulthood, functional studies have related prosocial behaviors, e.g. helping and sharing, to activity in regions involved in: (1) social cognition, e.g. medial prefrontal cortex (mPFC), tempo-parietal junction (TPJ), precuneus, superior temporal sulcus (STS; Schreuders et al., 2018; van der Meulen et al., 2016; Van Hoorn et al., 2016; Zanon et al., 2014), (2) behavioral control and decision making, e.g. dorso-lateral (dlPFC; Steinbeis et al., 2012; Telzer et al., 2011), and (3) empathy, e.g. anterior cingulate cortex (ACC) and anterior insula (AI; Hein et al., 2016; Hein et al., 2010). The ACC also contains Von Economo neurons, known to be involved in social cognition (Allman et al., 2005).

Two studies have examined prosociality and brain structure. In children aged 6-9 years, mostly positive associations were observed between prosociality and cortical thickness (CT) in regions involved in (1) social cognition, e.g. anterior temporal lobe, mPFC, cuneus and precuneus, (2) behavioral control, e.g. dlPFC, and (3) empathy, e.g. pars orbitalis (Thijssen et al., 2015; Wildeboer et al., 2017). These relations were predominantly identified in the right hemisphere for both males and females, except for a few regions where these associations were present in males alone (Thijssen et al., 2015). To our knowledge, no studies have examined prosocial behavior and structural change longitudinally across adolescence. As adolescence is a period characterized by changes in social cognition and peer relations, and marked maturation of associated brain structure and function (Foulkes and Blakemore, 2018), such longitudinal studies would arguably be highly informative for understanding the development of prosocial behaviors.

The aim of the current study was to examine how prosociality relates to the longitudinal development of CT in adolescence and young adulthood. Normative cortical development from childhood to adulthood is characterized by regionally variable decreases in CT as derived from MRI (Tamnes et al., 2017; Vijayakumar et al., 2016; Wierenga et al., 2014; Zhou et al., 2015). Here, we used the terms cortical thickness (CT) and cortical thinning, but it is in principle possible that the cortex in development is not thinning (further discussion in Supporting Information (S1.1)). Although thinning during development is not uniformly related to adaptive outcomes (Bos et al., 2018; Jirsaraie et al., 2018; Luby et al., 2018; Román et al., 2018), studies have associated greater cortical thinning in adolescence with reduced neuroticism and aggressive behavior, greater intellectual ability and more adaptive emotion regulation (Ferschmann et al., 2018; Shaw et al., 2006; Vijayakumar et al., 2014a, 2014b, 2014c). Moreover, delayed and slower rates of cortical maturation have been related to symptoms of anxiety and depression in healthy individuals (Ducharme et al., 2013), and ADHD (Shaw et al., 2011). Since the relations between CT trajectories and developmental outcomes may be regionally specific (Fandakova et al., 2017; Vijayakumar et al., 2014a) and since there is some evidence that the rate of CT change is not stable from childhood to adulthood (Zhou et al., 2015), it is possible that a crucial factor in explaining the effects of the rate of cortical thinning may be its timing.

Taken together, we therefore hypothesized that 1) higher levels of prosociality would be associated with greater age-expected thinning as many studies linked this developmental pattern to adaptive behaviors, and 2) that the associations would be region-specific. More specifically, given the role of social-cognitive and social-affective processes in prosociality, we also hypothesized that prosociality would be associated with regions implicated in ToM (Blakemore, 2012; Frith and Frith, 2003): mPFC, temporal poles, posterior STS, and TPJ, as well as regions related to empathy, i.e. AI and ACC (Lamm et al., 2011). Moreover, given the crucial role of behavior control in prosociality, we hypothesized that prosociality would be related to maturation of cortical regions associated with behavioral control (Hare et al., 2009; Steinbeis et al., 2014), i.e. dlPFC and ventromedial prefrontal (vmPFC) cortices.

\section{Methods}

\subsection{Participants}

The sample was drawn from NeuroCognitive Development (Tamnes et al., 2018, 2013; Tamnes et al., 2010), an accelerated longitudinal project with three waves, see Supporting Information (S2.1) for details. Table 1 describes the sample; 64 individuals provided data from one time point (TP), 78 from 2 TPs and 27 from 3 TPs.

In total 301 observations were available for individuals who had at least one TP with MRI data of sufficient quality (see 2.4) and selfreported prosocial behavior. For each individual, we included only those TPs where both MRI and prosociality data were available. Table 2 provides an overview of sample characteristics. Refer to Supporting Information for further detail, including attrition analyses (S2.1).

\subsection{Prosocial behavior}

Prosociality was assessed using the 5-item self-report prosocial subscale of the Strengths and Difficulties Questionnaire (Goodman, 1997). The items assessing different aspects of prosociality (e.g. "I usually share with others", "I often volunteer to help others") are rated on a 3-point Likert Scale as "not true", "somewhat true" and "certainly true". Mean inter-item correlation for this scale was 0.23 , a value within the recommended range (Briggs and Cheek, 1986). See Supporting Information (S2.2.1) for discussion of scale reliability. Mean prosocial score in this sample was $8.38(S D=1.41)$

\subsection{MRI acquisition}

The same 12-channel head coil on the same 1.5 T Siemens Avanto scanner (Siemens Medical Solutions, Erlangen, Germany) was used to collect MRI data at all 3 waves. The sequence used for morphological analyses at all three time points was a 3D T1-weighted magnetization prepared rapid gradient echo (MPRAGE), see parameters in Supporting Information (S2.3).

\subsection{MRI processing and acquisition}

Whole brain segmentation and cortical surface reconstruction was performed using FreeSurfer 6.0 (http://surfer.nmr.mgh.harvard.edu/)

Table 1

Demographics per data collection wave.

\begin{tabular}{|c|c|c|c|c|c|c|}
\hline & $\mathrm{N}$ & $\begin{array}{l}\text { Sex } \\
\text { Females/ } \\
\text { Males }\end{array}$ & $\begin{array}{l}\text { Age } \\
\text { Mean } \\
(S D)\end{array}$ & $\begin{array}{l}\text { Age } \\
\text { Min- } \\
\text { max }\end{array}$ & $\begin{array}{l}\text { Retained } \\
\text { from } \\
\text { previous } \\
\text { waves }\end{array}$ & $\begin{array}{l}\text { Newly } \\
\text { recruited }\end{array}$ \\
\hline $\begin{array}{c}\text { Wave } \\
1\end{array}$ & 101 & $53 / 48$ & $\begin{array}{l}15.8 \\
(2.35)\end{array}$ & $\begin{array}{l}12.0- \\
19.7\end{array}$ & - & 101 \\
\hline $\begin{array}{c}\text { Wave } \\
2\end{array}$ & 121 & $61 / 60$ & $\begin{array}{l}16.7 \\
(2.88)\end{array}$ & $\begin{array}{l}11.9- \\
21.9\end{array}$ & 61 & 60 \\
\hline $\begin{array}{c}\text { Wave } \\
3\end{array}$ & 106 & $58 / 48$ & $\begin{array}{l}20.1 \\
(3.27)\end{array}$ & $\begin{array}{l}13.8- \\
26.1\end{array}$ & 106 & 0 \\
\hline Total & 169 & $92 / 77$ & $\begin{array}{l}17.7 \\
(3.43)\end{array}$ & $\begin{array}{l}11.9- \\
26.1\end{array}$ & & \\
\hline
\end{tabular}

Note: New participants were recruited at wave 1 and wave 2. Colum "Retained from previous waves" refers to individuals who participated at a time point 2 (or 3 ) and also have data from wave 1 and/or 2 . 
Table 2

Additional sample demographics.

\begin{tabular}{lllll}
\hline & Wave 1 & Wave 2 & Wave 3 & All waves \\
\hline $\begin{array}{l}\text { Parental education } \\
\text { Parental education, }\end{array}$ & $3.2(0.71)$ & $3.3(0.69)$ & & $3.24(0.70)$ \\
$\quad 1-4$ & $1-4$ & & $1-4$ \\
$\quad$ range & $4.2(1.29)$ & $4.4(1.28)$ & & $4.28(1.26)$ \\
$\begin{array}{l}\text { Parental income } \\
\text { Parental income, range }\end{array}$ & $1-7$ & $1-7$ & & $1-7$ \\
WASI score & 110.8 & 111.2 & 112.9 & 111.6 \\
& $(10.6)$ & $(11.8)$ & $(10.3)$ & $(10.8)$ \\
WASI score, range & $88-141$ & $82-166$ & $86-139$ & $82-166$ \\
Years since last wave & & $2.6(0.17)$ & $4.2(0.33)$ & \\
\hline
\end{tabular}

Note: Values are means (standard deviations), unless otherwise specified. Parental education and income are based on values from both parents when available. New participants were recruited at wave 2 .

(Dale et al., 1999; Fischl, 2012; Fischl et al., 2002, 1999). CT, defined as the shortest distance between the gray/white matter boundary and the outer cortical boundary, was measured at each vertex across the surface. The longitudinal stream in FreeSurfer 6.0, used to deal with the longitudinal nature of the data, (Reuter et al., 2012) as well as MRI quality control procedures are described in Supporting Information (S2.3/S2.3.1). CT was down-sampled to the 81,924 vertices of fsaverage6, and surface maps were smoothed with a Gaussian kernel of full-width at half-maximum of $10 \mathrm{~mm}$.

\subsection{Statistical analyses}

\subsubsection{Prosocial behavior development}

Development of prosociality over time was assessed using the nmle package (Pinheiro et al., 2018) in $R$ (R Core Team, 2018), in Rstudio (www.rstudio.com). This and subsequent models that were tested in mixed-effects models where subject was entered as a random effect to account for within-individual dependence, i.e. responses from one individual over multiple TPs are likely to be correlated. Mixed-effects models deal with this form of dependency in the data, allow irregularly spaced measurements (Gibbons et al., 2010), and help to preserve power in case of missing data (Matuschek et al., 2017). We began by first identifying the best fitting development model for prosociality by comparing linear, quadratic, and cubic effects of age, as well as the null model. Model testing was performed using the likelihood ratio (LRT) test where models were fit by maximum likelihood (ML) estimation method. We considered both the LRT p-values and Akaike Information Criterion [AIC, (Akaike, 1974)] during model selection, and a more complex model was only chosen if $\mathrm{p}<0.05$ and AIC value indicated better fit (value that was smaller by at least two). In the next step, we investigated whether inclusion of sex improved the "developmental model", using the same model selection procedure. First, the main effect of sex was added to the model, followed by an interaction of age and sex. Model equations are outlined in Supporting Information (S2.4.1).

\subsubsection{Cortical thickness development}

Considering the lack of studies on prosociality and CT development, analyses were not limited to a priori selected regions of interests. Instead, whole-brain analyses across the cortical surface were performed to reduce the risk of Type II errors. Background analyses were conducted to assess the developmental trajectory of CT using Surfstat (http://www. math.mcgill.ca/keith/surfstat/), a toolbox created for MATLAB (The MathWorks, Inc., Nathan, MA). Random field theory (RFT) corrections ( $p<0.05$, cluster-defining threshold 0.005) were used to account for multiple comparisons (Worsley et al., 2004). The best-fit model for the developmental trajectory of CT was assessed in three steps whereby a cubic model was tested first, followed by a quadratic model, and a linear model (see (S2.4.2) for equations and (S3.1.1) for results).

\subsubsection{Associations between prosocial behavior and cortical thickness development}

As the background analyses of CT identified quadratic changes with age (see Supporting Information (S2.4.2 and S3.1.1)), we next tested interactions between prosociality and quadratic age terms using mixed models in SurfStat (see (S2.4.3) for equations). While linear mixed models are a valid approach for rejecting the null hypothesis that the relationship is linear, the quadratic function should not be interpreted literally as the shape of true developmental trajectory (Fjell et al., 2010). While inferring break points of age-trajectories based on quadratic models are heavily influenced by the age-range of the sample, a nonparametric smoothing approach seems to be more robust to such influences (Fjell et al., 2010). Thus, smoothing splines are recommended for interpreting the shapes of developmental trajectories. Therefore, we extracted average CT of significant clusters from the linear mixed model and used generalized additive mixed models (GAMM) with a cubic spline basis in R (Wood, 2006) to visualize developmental trajectories. This methodological choice is further elaborated in Supporting Information (S2.4.3). In a series of post hoc analyses, the model was rerun while controlling for estimated IQ, parental income, and parental education, see Supporting Information (S3.1.2).

\section{Results}

\subsection{Prosocial behavior development}

Results showed main effects of age and sex for prosocial behavior, but no interaction between age and sex (see Supplementary Tables 1 and 2 for model fit). There was an increase in prosocial behavior with age, and females reported themselves as more prosocial than males (Fig. 1).

\subsection{Prosocial behavior and cortical thickness development}

Analyses testing interactions between prosocial behavior and quadratic age-related cortical development yielded seven significant clusters within the following regions: 1 ) right posterior middle temporal cortex, 2) left dlPFC, 3) right inferior frontal gyrus (IFG), 4) right (d) $\mathrm{mPFC}, 5$ ) right intraparietal sulcus, 6 ) right posterior superior temporal cortex and TPJ, 7) right dorsal (d)ACC. The clusters are shown in Fig. 2 and further details are provided in Supplementary Table 3. Controlling for estimated IQ, parental education, and parental income did not substantially change the results (see Supporting Information (S3.1.2)).

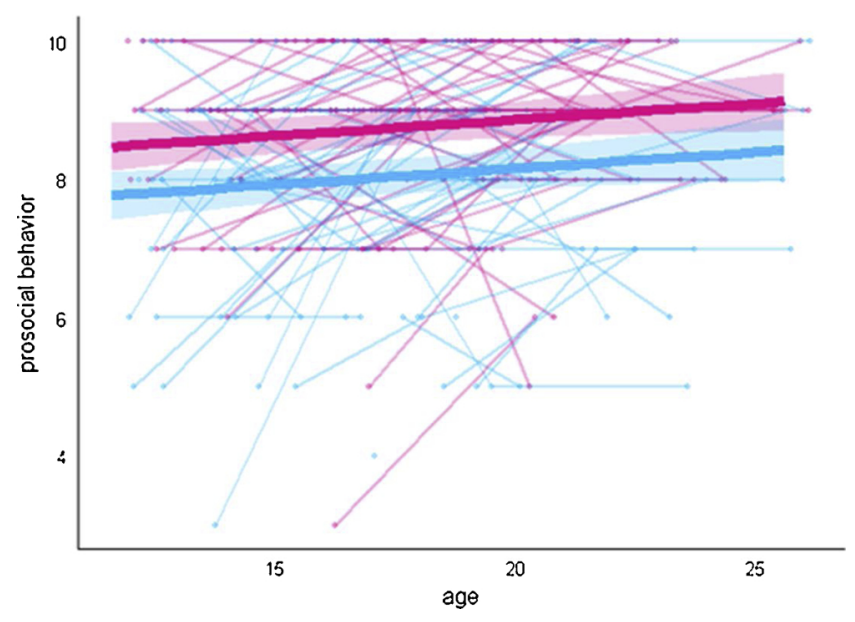

Fig. 1. Developmental trajectory for prosocial behavior. Females are represented in pink and males are represented in blue. The shaded areas correspond to the $95 \%$ confidence intervals. Significance testing was performed using likelihood ratio (LRT) test where models were fit by maximum likelihood (ML) estimation method. 

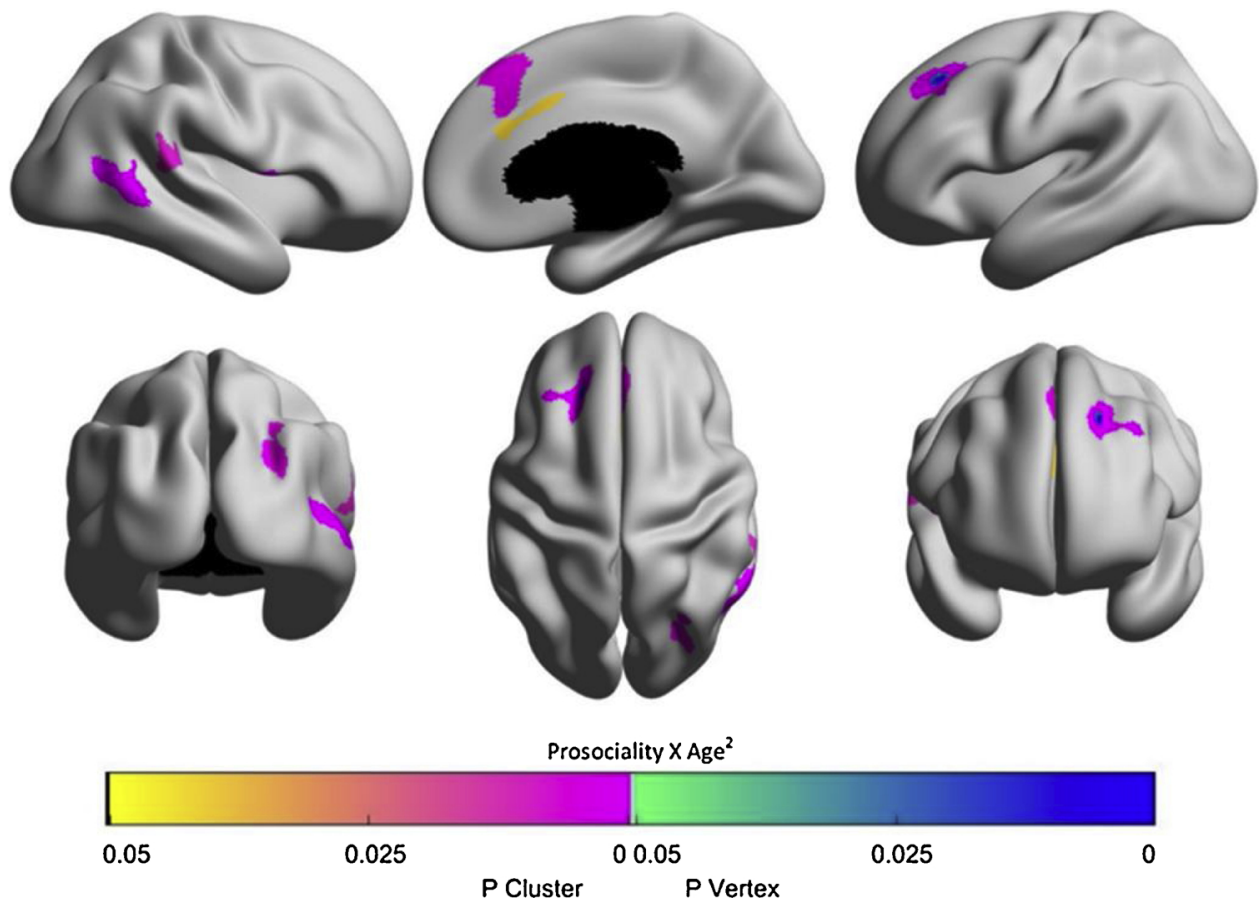

Fig. 2. Regions where interactions between prosociality and age ${ }^{2}$ on cortical thickness were significant. Yellow-pink regions represent cluster level p-value map, green-blue regions represent vertex level p-value maps; both $p \leq 0.05$, Random Field Theory (RFT) corrected. Note that the vertex-wise level correction is more stringent than cluster-based correction (Woo et al., 2014), thus resulting in less widespread effects.

To illustrate these relations between prosociality and CT development, participants were divided into two groups depending on their prosocial score throughout the study: stable low or stable high, see Supporting Information (S2.4.3). Cortical development was plotted for each group to estimate the developmental trajectories (Fig. 3). Higher scores on prosocial behavior were associated with greater thinning between early and mid-adolescence, which then slowed to less thinning during the transition to young adulthood. Lower scores on prosocial behavior showed the reverse pattern of slower thinning at younger ages and greater thinning at older ages. This pattern was observed across all the identified significant clusters. Note that the low/high division was made for illustration purposes only and the statistical analyses were run with prosocial behavior as a continuous measure.

To further probe the data, relations between prosociality, CT and age were visualized using contour plots (Fig. 4). The plots indicate that the relations between prosociality and CT were positive for the youngest and

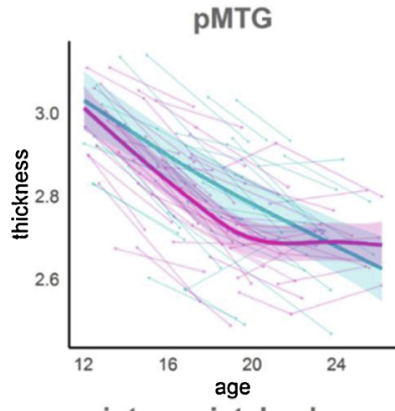

intraparietal sulcus

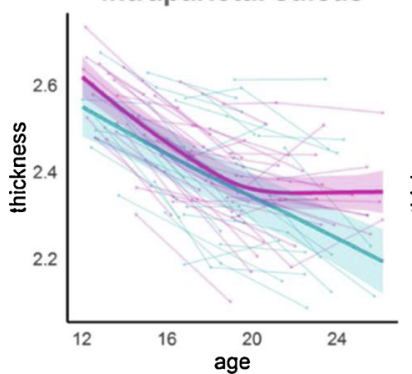

dIPFC

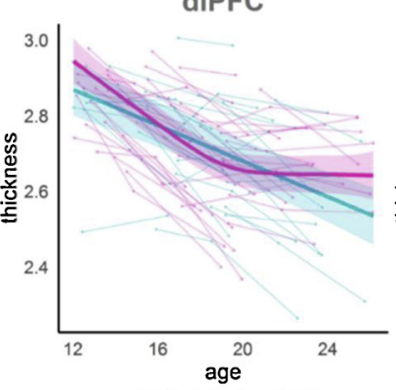

STG,TPJ,pSTS

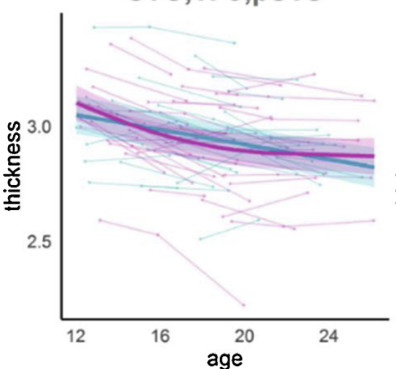

IFG
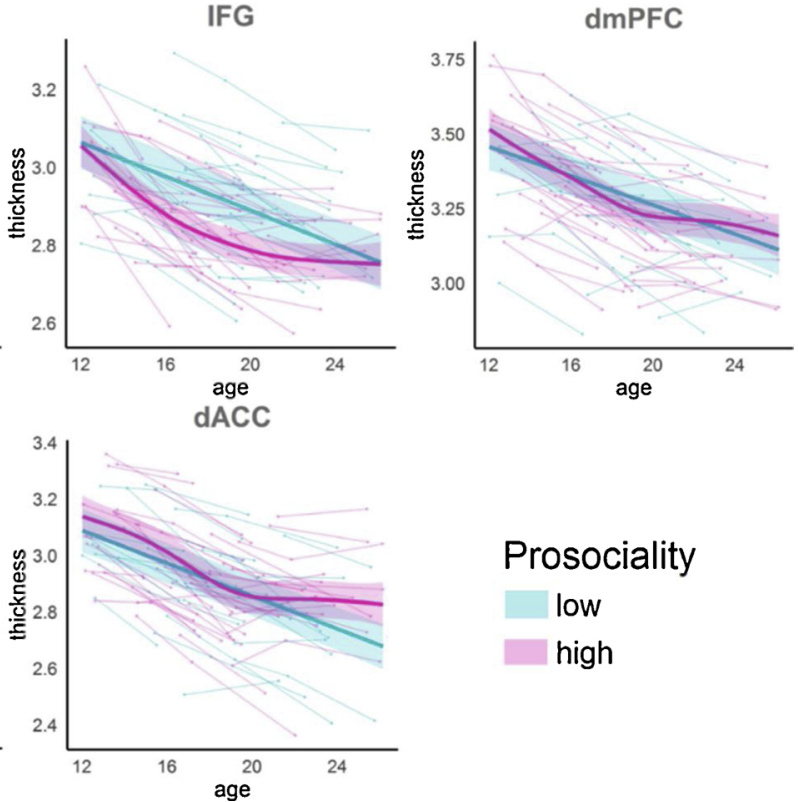

\section{Prosociality}

low

high

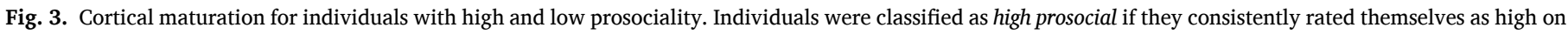

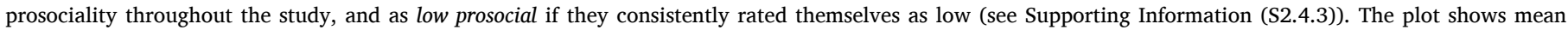

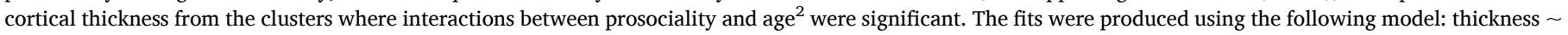

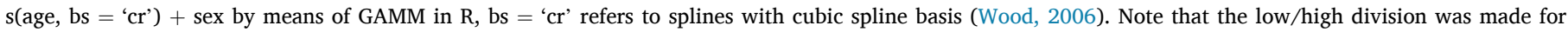
illustration purposes only, while the statistical analyses were run with prosocial behavior as a continuous measure. 
the oldest group across all the identified significant clusters; in the youngest group high prosocial scores were related to thicker cortex (white/light pink) and lower scores with thinner cortex (pink), the same pattern was found for the oldest group where high prosocial scores were associated with thicker cortex (yellow/light green) and low prosocial scores with thicker cortex (dark green).

\section{Discussion}

The current study examined the relations between prosociality and longitudinal CT development in adolescents and young adults. Higher self-reported prosociality was associated with greater initial rate of regional thinning during early adolescence, followed by attenuation of this process during the transition to young adulthood in regions involved in social cognition and behavior control. In contrast, lower prosocial scores were related to initially slower thinning, followed by more maturation into the third decade of life. These results suggest that the rate of thinning and possibly its timing, are key factors in prosociality during development.

Existing studies disagree on whether relatively greater thinning in development is adaptive (see e.g. (Bos et al., 2018; Vijayakumar et al., 2014a, 2014b, 2014c)). Similarly, being prosocial is generally considered to be positive, but being too prosocial may be maladaptive (Steinbeis, 2018). In our sample, prosociality was i) negatively related to a number of maladaptive developmental outcomes (see supplementary analyses (S3.2.2)), and ii) associated with greater thinning in early adolescence and more attenuated thinning in young adulthood. Although highly speculative, these results may imply that greater cortical thinning is adaptive in younger ages, but may no longer be optimal in young adulthood. In other words, greater thinning may or may not be optimal depending on age. However, this notion should be scrutinized by future studies. Previous studies have linked interventions promoting prosociality to number of favorable developmental outcomes (Caprara et al., 2014). Although highly speculative, future studies could test a hypothesis that such interventions during this time of plasticity may have lasting impacts through their effect on neural development.

Links between prosociality and longitudinal development of CT were identified in several regions and were in line with our hypotheses. First, anatomical locations previously associated with ToM, including TPJ, pSTS and posterior parts of MPFC, all in the right hemisphere were identified. The precise role of ToM in prosociality is not fully understood. Understanding one's mental states may directly lead to prosocial behaviors (Eggum et al., 2011), but it is also possible to use this ability for both good (prosocial behaviors) and ill (e.g. antisocial behavior such as manipulation). Therefore, ToM should rather be viewed as an information-gathering tool serving various purposes (Eisenberg et al., 2001). ToM may also be related to prosociality by affecting empathic concern (Van der Graaff et al., 2018), sympathy and moral reasoning (Eisenberg et al., 2001). The role of social approval has also been emphasized (Lane et al., 2010): individuals proficient in perspective taking have the ability to consider how others will react to their behavior, and anticipation of these reactions may motivate prosocial behaviors valued in one's social environment, e.g. doing the right thing to make others proud of you.

We also found an association between prosociality and cortical development in the right intraparietal sulcus, a part of the so-called mirror neuron system (MNS) in humans. This network is thought to aid understanding of others' actions and the intentions behind these actions (Cattaneo and Rizzolatti, 2009). Importantly, the MNS has been implicated in a longitudinal link between childhood empathy and prosociality in adolescence, albeit in more frontal components than in the current study (Flournoy et al., 2016). A large meta-analysis (Van Overwalle and Baetens, 2009) of functional neuroimaging studies suggests that the ToM and MNS represent two separate, but complementary neural networks, that both serve to optimize aspects of social processing. Consistently, our findings suggest that both networks may also contribute to prosociality.

Interestingly, these findings were located in the right hemisphere. There is some limited evidence of right hemispheric specialization for social emotions (Benowitz et al., 1983; Blonder et al., 1993), which may partially explain this finding. Additionally, Von Economo cells are more numerous in the right hemisphere (Allman et al., 2005), and studies on ToM have also emphasized this hemisphere (Scholz et al., 2009; Young et al., 2010).

While systems supporting understanding others' actions, intentions, thoughts and feelings, may be important contributors to prosociality, systems supporting strategic decision-making may also be critical. Prosocial behaviors are vital for living in social groups, an arrangement that has been beneficial for humans throughout evolution, e.g. advantages such as resource sharing or group protection. Groups with larger number of prosocial individuals are more likely to succeed over groups consisting mainly of selfish individuals, and therefore, at least under some
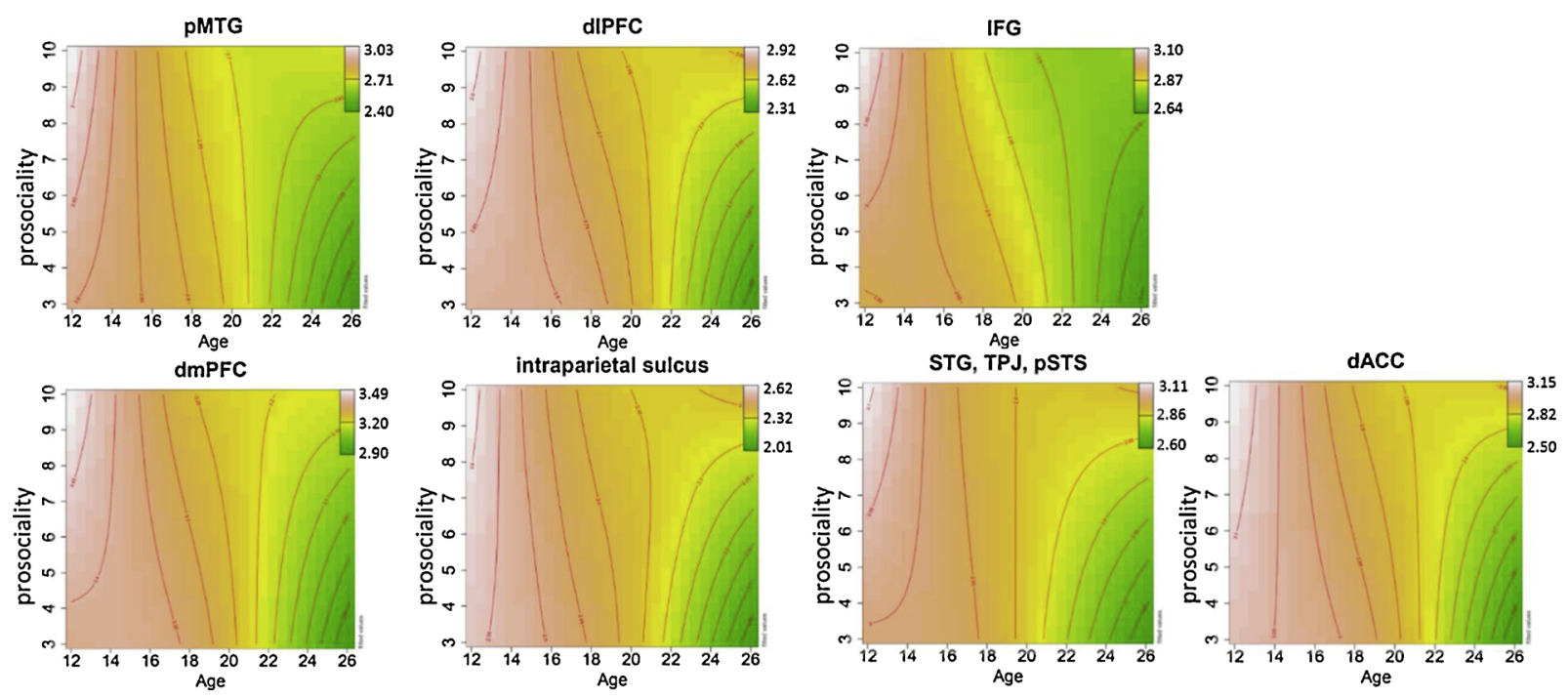

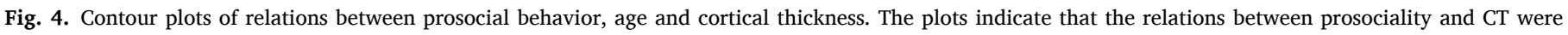

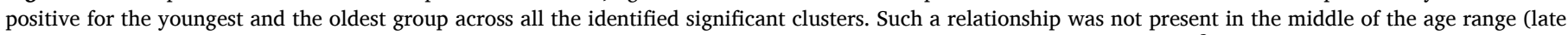

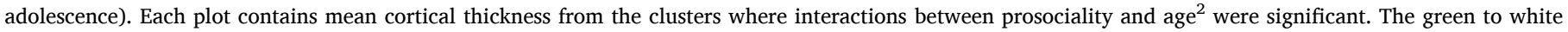
color scale indicates low to high cortical thickness values. 
circumstances, evolutionary mechanisms may have contributed to the emergence of prosocial behaviors in social groups (see Van Vugt and van Lange, 2006 for review). Not acting in a prosocial manner may cause retaliation and exclusion by group members (Axelrod and Hamilton, 1981; Fehr and Gachter, 2000). On the other hand, acting excessively prosocially can put a strain on one's resources and may place one in at a disadvantage in some situations. Considering these costs and benefits of being prosocial prior to engagement in such acts may thus be adaptive (Steinbeis, 2018). Our results showed that prosociality was associated with CT development in the left dlPFC, but not vmPFC, despite both regions supporting value-based decision-making (Sokol-Hessner et al., 2012). The left dlPFC has previously been identified in behavioral control and implicated in strategic social behavior (Steinbeis et al., 2012), and as a key structure involved in self-control (Hare et al., 2009), a function that impacts decisions to act prosocially (Joosten et al., 2015; Osgood and Muraven, 2015). Additionally, transcranial stimulation of this region has been associated with increased prosociality (Balconi and Canavesio, 2014), and bilateral dlPFC activation has been associated with sharing in the context of social norm compliance (Spitzer et al., 2007). Taken together, prior findings implicate the dlPFC supporting the integration of emotional information in decision making in settings (Balconi and Canavesio, 2014), thus highlighting the potential role of this structure in prosocial behaviors.

In line with our hypotheses, prosociality was also related to CT development of the right AACC, as region that has repeatedly been linked to cognitive control, performance-monitoring, motivation and reward-based decision-making (see Shenhav et al., 2016 for review). The overall expected value of control theory (EVC) integrates these findings and proposes that the AACC is involved in making decisions as to how much control is allocated based on cost/benefit analysis of estimated reward outcome and effort cost (Shenhav et al., 2016). This is in line with the notion that prosocial behaviors depend on thorough situational assessment and cost-benefit analysis. Additionally, this phylogenetically and ontogenetically late developing structure has repeatedly been implicated in empathy (Hein et al., 2016; Hein et al., 2010) and contains Von Economo cells (Allman et al., 2005) that are involved in rapid assessment of complex situations and provide necessary input to regions involved in ToM.

Prior research on samples aged 6-9, have identified predominantly positive associations between prosociality and CT (Thijssen et al., 2015; Wildeboer et al., 2017). Our study differed by focusing on an older age-range, 12-26 years, and by employing a longitudinal design to test for associations with cortical development (i.e. changes in CT over time). Still, some of the same regions in superior medial and lateral frontal cortices were implicated in our study as well as by Thijssen et al. (2015) who used the same measure of prosociality. In line with these earlier findings, we also observed positive associations in the younger age range of our sample (see Fig. 4). Additionally, previous work on symptoms of $\mathrm{CD}$ based on data from the first wave of the current project found negative relations between symptoms of CD and CT, with the relation being strongest in the youngest individuals (Walhovd et al., 2012). Prosocial and antisocial behaviors have by some been conceptualized as extreme ends of a continuum (Eron and Huesmann, 1984), although the relation is likely more complex (see Eisenberg et al., 2015 for review). Nonetheless, Waldman et al. (2011) uncovered an overlap between genetic influences on $\mathrm{CD}$ and prosociality, and similar areas involved in regulatory processes and social-cognitive processing have been implicated in brain structural studies (see Walhovd et al., 2012). Considering our longitudinal and the cross-sectional results together, although speculative, it is possible that highly prosocial individuals differ from less prosocial individuals by having thicker cortices to begin with in early development, followed by differential pattern of thinning into young adulthood.

While not a primary focus of this study, we found that prosociality showed subtle linear increases from age 12 to young adulthood. This is largely consistent with prior literature that has shown decreases in early adolescence followed by a rebound (Eisenberg et al., 2015). However, we failed to identify any associations between change in prosociality and changes in CT over adolescence, which likely relates to the moderate levels of rank-order stability in prosocial behaviors (see S3.2.1). Females also reported being more prosocial but the rate of increase over time was identical for both sexes. This is in line with existing research, demonstrating less prosociality in males, particularly when self-report measurement is used (Fabes and Eisenberg, 1998). These differences may reflect conceptions of what males and females are supposed to be like, rather than actual behaviors. Additionally, measures of prosocial behavior may include a disproportionate number of items on which girls are more likely to rate themselves as prosocial than boys (see (Fabes and Eisenberg, 1998) for review. However, we note that interactions between cortical development and prosocial behavior were not driven by these sex differences (see Supporting Information (S3.2.6) for further detail).

Future studies should also employ more specific measures of prosociality as different subtypes of prosociality, i.e. helping, sharing and comforting, may depend on different forms of social-cognitive understanding and show unique developmental trajectories (Dunfield, 2014). They may thus also relate to distinct cortical regions and patterns of cortical development. Self-report used in the current study should be supplemented by additional measures of prosociality provided by different observers in order to reduce the impact of social-desirability bias which may have influenced results in this study. Additionally, psychometric properties of the SDQ have not been tested for the entire age-range of the current sample. While this may constitute a possible limitation, the continuity of measures across time points was considered to be more important. Future studies could also compare cortical maturation in highly antisocial individuals to that of highly prosocial individuals to examine whether there is support for a dimensional view of prosocial-antisocial behaviors. Moreover, note that estimates of non-linear development in our study were driven by between-subject differences. Future studies with more time points are needed to model within-subject non-linear trajectories. Finally, future studies should include measures of pubertal maturation, a key factor driving individual differences in brain structural development (Herting and Sowell, 2017). This may provide further insight into the relations between cortical development and prosocial behavior.

In conclusion, the longitudinal nature of the current study offers novel insight into the emergence of the links between prosociality and brain structure, specifically highlighting the moderating role of age; the association changed from positive to negative across early to late adolescence, and subsequently became positive again in young adulthood. These dynamic relations were observed in regions previously associated with social processing and behavioral control. The results emphasize that both the rate and the timing of cortical thinning may be related to prosocial behaviors during development. Given the study's exploratory nature and limitations, the conclusions are speculative and should be interpreted with caution

\section{Funding}

This study was supported by the Research Council of Norway (to AMF, KBW and CKT: 230345, 271839, 288083, 223273)

\section{Declaration of Competing Interest}

No conflict of interest

\section{Appendix A. Supplementary data}

Supplementary material related to this article can be found, in the online version, at doi:https://doi.org/10.1016/j.dcn.2019.100734. 


\section{References}

Allman, J.M., Watson, K.K., Tetreault, N.A., Hakeem, A.Y., 2005. Intuition and autism: a possible role for Von Economo neurons. Trends Cogn. Sci. 9 (8), 367-373.

Axelrod, R., Hamilton, W.D., 1981. The evolution of cooperation. Science 211 (4489), 1390-1396.

Balconi, M., Canavesio, Y., 2014. High-frequency rTMS on DLPFC increases prosocial attitude in case of decision to support people. Soc. Neurosci. 9 (1), 82-93.

Benowitz, L.I., Bear, D.M., Rosenthal, R., Mesulam, M.M., Zaidel, E., Sperry, R.W., 1983 Hemispheric specialization in nonverbal communication. Cortex 19 (1), 5-11.

Blakemore, S.-J., 2012. Development of the social brain in adolescence. J. R. Soc. Med. 105 (3), 111-116

Blonder, L.X., Burns, A.F., Bowers, D., Moore, R.W., Heilman, K.M., 1993. Right hemisphere facial expressivity during natural conversation. Brain Cogn. 21 (1), 44-56.

Bos, M.G., Peters, S., van de Kamp, F.C., Crone, E.A., Tamnes, C.K., 2018. Emerging depression in adolescence coincides with accelerated frontal cortical thinning. J. Child Psychol. Psychiatry.

Briggs, S.R., Cheek, J.M., 1986. The role of factor analysis in the development and evaluation of personality scales. J. Pers. 54 (1), 106-148.

Caprara, G.V., Barbaranelli, C., Pastorelli, C., Bandura, A., Zimbardo, P.G., 2000 Prosocial foundations of children's academic achievement. Psychol. Sci. 11 (4), 302-306.

Caprara, G.V., Kanacri, B.P.L., Gerbino, M., Zuffiano, A., Alessandri, G., Vecchio, G., et al., 2014. Positive effects of promoting prosocial behavior in early adolescence: Evidence from a school-based intervention. Int. J. Behav. Dev. 38 (4), 386-396.

Caputi, M., Lecce, S., Pagnin, A., Banerjee, R., 2012. Longitudinal effects of theory of mind on later peer relations: the role of prosocial behavior. Dev. Psychol. 48 (1), $257-270$.

Cattaneo, L., Rizzolatti, G., 2009. The mirror neuron system. Arch. Neurol. 66 (5), $557-560$

Dale, A.M., Fischl, B., Sereno, M.I., 1999. Cortical surface-based analysis: I. Segmentation and surface reconstruction. Neuroimage 9 (2), 179-194.

Ducharme, S., Albaugh, M.D., Hudziak, J.J., Botteron, K.N., Nguyen, T.-V., Truong, C., et al., 2013. Anxious/depressed symptoms are linked to right ventromedial prefrontal cortical thickness maturation in healthy children and young adults. Cereb. Cortex 24 (11), 2941-2950.

Dunfield, K.A., 2014. A construct divided: prosocial behavior as helping, sharing, and comforting subtypes. Front. Psychol. 5, 1-12.

Eggum, N.D., Eisenberg, N., Kao, K., Spinrad, T.L., Bolnick, R., Hofer, C., et al., 2011. Emotion understanding, theory of mind, and prosocial orientation: relations over time in early childhood. J. Posit. Psychol. 6 (1), 4-16.

Eisenberg, N., Guthrie, I.K., Cumberland, A., Murphy, B.C., Shepard, S.A., Zhou, Q., Carlo, G., 2002. Prosocial development in early adulthood: a longitudinal study. J. Pers. Soc. Psychol. 82 (6), 993-1006.

Eisenberg, N., Spinrad, T.L., Knafo-Noam, A., 2015. Prosocial development. In: Lerner, R. M. (Ed.), Handbook of Child Psychology and Developmental Science, Vol. 3. John Wiley \& Sons, New Jersey.

Eisenberg, N., Zhou, Q., Koller, S., 2001. Brazilian adolescents' prosocial moral judgment and behavior: relations to sympathy, perspective taking, gender-role orientation, and demographic characteristics. Child Dev. 72 (2), 518-534.

Eron, L.D., Huesmann, L.R., 1984. The relation of prosocial behavior to the development of aggression and psychopathology. Aggress. Behav. 10 (3), 201-211.

Fabes, R.A., Eisenberg, N., 1998. Meta-Analyses of Age and Sex Differences in Children's and Adolescents' Prosocial Behavior. Working paper, Arizona State, Tempe, AZ.

Fandakova, Y., Selmeczy, D., Leckey, S., Grimm, K.J., Wendelken, C., Bunge, S.A. Ghetti, S., 2017. Changes in ventromedial prefrontal and insular cortex support the development of metamemory from childhood into adolescence. Proc. Natl. Acad. Sci. 114 (29), 7582-7587.

Fehr, E., Gachter, S., 2000. Cooperation and punishment in public goods experiments. Am. Econ. Rev. 90 (4), 980-994.

Ferschmann, L., Fjell, A.M., Vollrath, M.E., Grydeland, H., Walhovd, K.B., Tamnes, C.K., 2018. Personality traits are associated with cortical development across adolescence: a longitudinal structural MRI study. Child Dev. 89 (3), 811-822.

Fischl, B., 2012. FreeSurfer. Neuroimage 62 (2), 774-781.

Fischl, B., Salat, D.H., Busa, E., Albert, M., Dieterich, M., Haselgrove, C., et al., 2002 Whole brain segmentation: automated labeling of neuroanatomical structures in the human brain. Neuron 33 (3), 341-355.

Fischl, B., Sereno, M.I., Dale, A.M., 1999. Cortical surface-based analysis: II: inflation, flattening, and a surface-based coordinate system. Neuroimage 9 (2), 195-207.

Flournoy, J.C., Pfeifer, J.H., Moore, W.E., Tackman, A.M., Masten, C.L., Mazziotta, J.C., et al., 2016. Neural reactivity to emotional faces may mediate the relationship between childhood empathy and adolescent prosocial behavior. Child Dev. 87 (6), 1691-1702.

Foulkes, L., Blakemore, S.-J., 2018. Studying individual differences in human adolescent brain development. Nat. Neurosci. 21, 315-323.

Frith, U., Frith, C.D., 2003. Development and neurophysiology of mentalizing. Philos. Trans. R. Soc. B: Biol. Sci. 358 (1431), 459-473.

Gibbons, R.D., Hedeker, D., DuToit, S., 2010. Advances in analysis of longitudinal data. Annu. Rev. Clin. Psychol. 6, 79-107.

Goodman, R., 1997. The strengths and difficulties questionnaire: a research note. J. Child Psychol. Psychiatry 38 (5), 581-586.

Hare, T.A., Camerer, C.F., Rangel, A., 2009. Self-control in decision-making involves modulation of the vmPFC valuation system. Science 324 (5927), 646-648.
Hay, D.F., Hudson, K., Liang, W., 2010. Links between preschool children's prosocial skills and aggressive conduct problems: the contribution of ADHD symptoms. Early Child. Res. Q. 25 (4), 493-501.

Hein, G., Morishima, Y., Leiberg, S., Sul, S., Fehr, E., 2016. The brain's functional network architecture reveals human motives. Science 351 (6277), 1074-1078.

Hein, G., Silani, G., Preuschoff, K., Batson, C.D., Singer, T., 2010. Neural responses to ingroup and outgroup members' suffering predict individual differences in costly helping. Neuron 68 (1), 149-160.

Herting, M.M., Sowell, E.R., 2017. Puberty and structural brain development in humans. Front. Neuroendocrinol. 44, 122-137.

Imuta, K., Henry, J.D., Slaughter, V., Selcuk, B., Ruffman, T., 2016. Theory of mind and prosocial behavior in childhood: a meta-analytic review. Dev. Psychol. 52 (8), 1192.

Jirsaraie, R., Rush, S., Kaczkurkin, A., Rosen, A., Sotiras, A., Ciric, R., et al., 2018. S139. Accelerated cortical thinning within structural brain networks is associated with irritability in youth. Biol. Psychiatry 83 (9), S402.

Joosten, A., van Dijke, M., Van Hiel, A., De Cremer, D., 2015. Out of control!? How loss of self-control influences prosocial behavior: the role of power and moral values. PLoS One 10 (5), e0126377.

Kuhnert, R.-L., Begeer, S., Fink, E., de Rosnay, M., 2017. Gender-differentiated effects of theory of mind, emotion understanding, and social preference on prosocial behavior development: a longitudinal study. J. Exp. Child Psychol. 154, 13-27.

Lamm, C., Decety, J., Singer, T., 2011. Meta-analytic evidence for common and distinct neural networks associated with directly experienced pain and empathy for pain. Neuroimage 54 (3), 2492-2502.

Lane, J.D., Wellman, H.M., Olson, S.L., LaBounty, J., Kerr, D.C., 2010. Theory of mind and emotion understanding predict moral development in early childhood. Br. J. Dev. Psychol. 28 (4), 871-889.

Luby, J.L., Agrawal, A., Belden, A., Whalen, D., Tillman, R., Barch, D.M., 2018. Developmental trajectories of the orbitofrontal cortex and anhedonia in middle childhood and risk for substance use in adolescence in a longitudinal sample of depressed and healthy preschoolers. Am. J. Psychiatry 4 (3), e4869.

Matuschek, H., Kliegl, R., Vasishth, S., Baayen, H., Bates, D., 2017. Balancing Type I error and power in linear mixed models. J. Mem. Lang. 94, 305-315.

Nowak, M.A., 2006. Five rules for the evolution of cooperation. science 314 (5805), 1560-1563.

Osgood, J.M., Muraven, M., 2015. Self-control depletion does not diminish attitudes about being prosocial but does diminish prosocial behaviors. Basic Appl. Soc. Psych. 37 (1), 68-80.

Pinheiro, J., Bates, D., DebRoy, S., Sarkar, D., R Core Team, 2018. Nlme: Linear and Nonlinear Mixed Effects Models. R Package Version 3.1-137 (Version 3.1-137). Retrieved from. https://CRAN.R-project.org/package=nlme.

Preckel, K., Kanske, P., Singer, T., 2018. On the interaction of social affect and cognition: empathy, compassion and theory of mind. Curr. Opin. Behav. Sci. 19, 1-6.

R Core Team, 2018. R: A Language and Environment for Statistical Computing. Vienna, Austria: R Foundation for Statistical Computing. Retrieved from. https://www.R-pro ject.org/.

Reuter, M., Schmansky, N.J., Rosas, H.D., Fischl, B., 2012. Within-subject template estimation for unbiased longitudinal image analysis. Neuroimage 61 (4), 1402-1418.

Román, F.J., Morillo, D., Estrada, E., Escorial, S., Karama, S., Colom, R., 2018. Brainintelligence relationships across childhood and adolescence: a latent-variable approach. Intelligence 68, 21-29.

Scholz, J., Triantafyllou, C., Whitfield-Gabrieli, S., Brown, E.N., Saxe, R., 2009. Distinct regions of right temporo-parietal junction are selective for theory of mind and exogenous attention. PLoS One 4 (3), e4869.

Schreuders, E., Klapwijk, E.T., Will, G.-J., Güroğlu, B., 2018. Friend versus foe: neura correlates of prosocial decisions for liked and disliked peers. Cogn. Affect. Behav. Neurosci. 1-16.

Shaw, P., Gilliam, M., Liverpool, M., Weddle, C., Malek, M., Sharp, W., et al., 2011. Cortical development in typically developing children with symptoms of hyperactivity and impulsivity: support for a dimensional view of attention deficit hyperactivity disorder. Am. J. Psychiatry 168 (2), 143-151.

Shaw, P., Greenstein, D., Lerch, J., Clasen, L., Lenroot, R., Gogtay, N., et al., 2006. Intellectual ability and cortical development in children and adolescents. Nature 440 (7084), 676.

Shenhav, A., Cohen, J.D., Botvinick, M.M., 2016. Dorsal anterior cingulate cortex and the value of control. Nat. Neurosci. 19 (10), 1286-1291.

Sokol-Hessner, P., Hutcherson, C., Hare, T., Rangel, A., 2012. Decision value computation in DLPFC and VMPFC adjusts to the available decision time. Eur. J. Neurosci. 35 (7), 1065-1074.

Spitzer, M., Fischbacher, U., Herrnberger, B., Grön, G., Fehr, E., 2007. The neural signature of social norm compliance. Neuron 56 (1), 185-196.

Steinbeis, N., 2018. Neurocognitive mechanisms of prosociality in childhood. Curr. Opin. Psychol. 20, 30-34.

Steinbeis, N., Bernhardt, B.C., Singer, T., 2012. Impulse control and underlying functions of the left DLPFC mediate age-related and age-independent individual differences in strategic social behavior. Neuron 73 (5), 1040-1051.

Steinbeis, N., Haushofer, J., Fehr, E., Singer, T., 2014. Development of behavioral control and associated vmPFC-DLPFC connectivity explains children's increased resistance to temptation in intertemporal choice. Cereb. Cortex 26 (1), 32-42.

Tamnes, C.K., Herting, M.M., Goddings, A.-L., Meuwese, R., Blakemore, S.-J., Dahl, R.E., et al., 2017. Development of the cerebral cortex across adolescence: a multisample study of inter-related longitudinal changes in cortical volume, surface area, and thickness. J. Neurosci. 37 (12), 3402-3412. 
Tamnes, C.K., Overbye, K., Ferschmann, L., Fjell, A.M., Walhovd, K.B., Blakemore, S.-J., Dumontheil, I., 2018. Social perspective taking is associated with self-reported prosocial behavior and regional cortical thickness across adolescence. Dev. Psychol.

Tamnes, C.K., Walhovd, K.B., Dale, A.M., Østby, Y., Grydeland, H., Richardson, G., et al., 2013. Brain development and aging: overlapping and unique patterns of change. Neuroimage 68, 63-74.

Tamnes, C.K., Østby, Y., Fjell, A.M., Westlye, L.T., Due-Tønnessen, P., Walhovd, K.B., 2010. Brain maturation in adolescence and young adulthood: regional age-related changes in cortical thickness and white matter volume and microstructure. Cereb. Cortex 20 (3), 534-548.

Telzer, E.H., Masten, C.L., Berkman, E.T., Lieberman, M.D., Fuligni, A.J., 2011. Neural regions associated with self control and mentalizing are recruited during prosocial behaviors towards the family. Neuroimage 58 (1), 242-249.

Thijssen, S., Wildeboer, A., Muetzel, R.L., Bakermans-Kranenburg, M.J., El Marroun, H., Hofman, A., et al., 2015. Cortical thickness and prosocial behavior in school-age children: apopulation-based MRI study. Soc. Neurosci. 10 (6), 571-582.

Tusche, A., Böckler, A., Kanske, P., Trautwein, F.-M., Singer, T., 2016. Decoding the charitable brain: empathy, perspective taking, and attention shifts differentially predict altruistic giving. J. Neurosci. 36 (17), 4719-4732.

Van der Graaff, J., Carlo, G., Crocetti, E., Koot, H.M., Branje, S., 2018. Prosocial behavior in adolescence: gender differences in development and links with empathy. J. Youth Adolesc. 47 (5), 1086-1099.

van der Meulen, M., van IJzendoorn, M.H., Crone, E.A., 2016. Neural correlates of prosocial behavior: compensating social exclusion in a four-player cyberball game. PLoS One 11 (7), e0159045.

Van Hoorn, J., Van Dijk, E., Güroğlu, B., Crone, E.A., 2016. Neural correlates of prosocial peer influence on public goods game donations during adolescence. Soc. Cogn. Affect. Neurosci. 11 (6), 923-933.

Van Overwalle, F., Baetens, K., 2009. Understanding others' actions and goals by mirror and mentalizing systems: a meta-analysis. Neuroimage 48 (3), 564-584.

Van Vugt, M., van Lange, P.A.M., 2006. The altruism puzzle : psychological adaptations for prosocial behavior. In: Schaller, M., Kenrick, D.T., Simpson, J.A. (Eds.), Evolution and Social Psychology. Frontiers of Social Psychology. Psychology Press Ltd, United Kingdom, pp. 237-262.

Vijayakumar, N., Allen, N.B., Youssef, G., Dennison, M., Yücel, M., Simmons, J.G., Whittle, S., 2016. Brain development during adolescence: a mixed-longitudinal investigation of cortical thickness, surface area, and volume. Hum. Brain Mapp. 37 (6), 2027-2038.
Vijayakumar, N., Whittle, S., Dennison, M., Yücel, M., Simmons, J., Allen, N.B., 2014. Development of temperamental effortful control mediates the relationship between maturation of the prefrontal cortex and psychopathology during adolescence: a 4year longitudinal study. Dev. Cogn. Neurosci. 9, 30-43.

Vijayakumar, N., Whittle, S., Yücel, M., Dennison, M., Simmons, J., Allen, N.B., 2014. Prefrontal structural correlates of cognitive control during adolescent development: a 4-year longitudinal study. J. Cogn. Neurosci. 26 (5), 1118-1130.

Vijayakumar, N., Whittle, S., Yücel, M., Dennison, M., Simmons, J., Allen, N.B., 2014. Thinning of the lateral prefrontal cortex during adolescence predicts emotion regulation in females. Soc. Cogn. Affect. Neurosci. 9 (11), 1845-1854.

Waldman, I.D., Tackett, J.L., Van Hulle, C.A., Applegate, B., Pardini, D., Frick, P.J., Lahey, B.B., 2011. Child and adolescent conduct disorder substantially shares genetic influences with three socioemotional dispositions. J. Abnorm. Psychol. 120 (1), 57.

Walhovd, K.B., Tamnes, C.K., Østby, Y., Due-Tønnessen, P., Fjell, A.M., 2012. Normal variation in behavioral adjustment relates to regional differences in cortical thickness in children. Eur. Child Adolesc. Psychiatry 21 (3), 133-140.

Warneken, F., 2016. Insights into the biological foundation of human altruistic sentiments. Curr. Opin. Psychol. 7, 51-56.

Wierenga, L.M., Langen, M., Oranje, B., Durston, S., 2014. Unique developmental trajectories of cortical thickness and surface area. Neuroimage 87, 120-126.

Wildeboer, A., Thijssen, S., Muetzel, R.L., Bakermans-Kranenburg, M.J., Tiemeier, H., White, T., Van IJzendoorn, M.H., 2017. Neuroanatomical correlates of donating behavior in middle childhood. Soc. Neurosci. 1-12.

Woo, C.-W., Krishnan, A., Wager, T.D.J.N., 2014. Cluster-extent based thresholding in fMRI analyses: pitfalls and recommendations. Neuroimage. 91, 412-419.

Wood, S.N., 2006. Generalized Additive Models: an Introduction With R. Chapman and Hall/CRC.

Worsley, K.J., Taylor, J.E., Tomaiuolo, F., Lerch, J., 2004. Unified univariate and multivariate random field theory. Neuroimage 23, S189-S195.

Young, L., Camprodon, J.A., Hauser, M., Pascual-Leone, A., Saxe, R., 2010. Disruption of the right temporoparietal junction with transcranial magnetic stimulation reduces the role of beliefs in moral judgments. Proc. Natl. Acad. Sci. 107 (15), 6753-6758.

Zanon, M., Novembre, G., Zangrando, N., Chittaro, L., Silani, G., 2014. Brain activity and prosocial behavior in a simulated life-threatening situation. Neuroimage 98 , 134-146.

Zhou, D., Lebel, C., Treit, S., Evans, A., Beaulieu, C., 2015. Accelerated longitudinal cortical thinning in adolescence. Neuroimage 104, 138-145. 